\title{
Les Journées de la Matière Condensée 2018
}

La division de physique de la Matière Condensée de la Société Française de Physique (SFP) assure le lien entre toutes les communautés étudiant les propriétés physiques des liquides et des solides, qu'ils soient cristallins ou amorphes, aux échelles allant du nanomètre à celles du monde qui nous entoure. Elle regroupe des domaines aussi divers que la physique quantique, l'étude des surfaces, la structure et la dynamique des liquides et des verres, les transitions de phase, la nanotechnologie, l'interaction rayonnement-matière, les polymères, les cristaux liquides.. Les interfaces thématiques y sont nombreuses, par exemple avec la physico-chimie, la mécanique, la biophysique...

Cette division organise depuis trente ans les Journées de la Matière Condensée (JMC). Ces journées ont lieu les années paires, en alternance avec le Congrès général de la SFP qui se tient les années impaires. La qualité des intervenants, la pertinence des sujets abordés, en particulier à travers les minicolloques, et l'interdisciplinarité, font des JMC l'un des plus grands congrès de physique en France et l'une des plus grandes rencontres de la physique de la matière condensée. Ces journées constituent un moment essentiel dans la vie scientifique de cette communauté et rencontrent toujours un grand succès, succès confirmé lors de sa $16^{\mathrm{e}}$ édition qui a rassemblé du 27 au 31 août 2018, sur le campus de l'Université Grenoble Alpes, environ 700 participants venus de toute la France et du monde francophone.

L'origine de cette réussite réside dans un programme très riche, avec un panel de 25 orateurs pléniers ou semi-pléniers de tout premier plan, 33 minicolloques proposés et organisés par la communauté. Tous mettent en lumière les sujets phares en matière condensée et contribuent à l'interdisciplinarité au travers d'interventions orales et de posters (150).

Cette année, l'accent a également été mis sur la place des femmes en science. Une table ronde plénière «Égalité des chances : un enjeu pour tous, hommes et femmes » organisée par la commission Femmes et Physique de la SFP et l'association Parité Sciences à Grenoble, a permis de débattre autour de deux thèmes : visibilité et harcèlement. Deux autres tables rondes semiplénières, ayant pour thèmes « Recherche et innovation en entreprise et emploi des docteurs 》 et le «Peer-review au $21^{\mathrm{e}}$ siècle », ont aussi été très suivies.

II faut souligner ici l'effort particulier de l'équipe organisatrice pour attirer des femmes et des jeunes venant d'horizons géographiques divers. En prenant le parti de discuter d'abord des femmes qu'ils aimeraient inviter, puis de choisir des hommes permettant d'obtenir un bon équilibre thématique, $40 \%$ des présentations plénières et semi-plénières ont été données par des femmes. Par ailleurs, des instructions précises envoyées aux organisateurs des minicolloques, rappelant notamment que les JMC étaient une occasion pour les jeunes de présenter leur travail, ont permis d'atteindre un peu plus de $50 \%$ de jeunes intervenants dans ces minicolloques et près de $70 \%$ pour les posters.

Mentionnons, pour terminer ce tour d'horizon, qu'en marge de ces journées le prix Charpak-Ritz, conjoint entre la SFP et la SPS (Société Suisse de Physique), et le prix Holweck, conjoint entre la SFP et I'IOP (Institute of Physics britannique), ont été remis respectivement à Roland Horisberger et Marina Galand. Ces grands prix de la SFP sont une excellente occasion de discuter avec les représentant.e.s de nos sociétés sœurs et d'examiner les actions communes possibles. Les prix Ancel 2016 et 2017 de la division Matière Condensée ont également été remis, avec pour lauréats Sara Ducci et Éric Collet.

Au final, les échanges, le brassage d'idées, la convivialité ont été possibles grâce au soutien de nos partenaires, conférenciers, exposants, bénévoles, et aux congressistes que nous remercions ici très chaleureusement. Le rendez-vous est d'ores et déjà pris dans deux ans, à Rennes, pour une nouvelle édition des JMC !

Catherine Langlais ${ }^{(1)}$, Laëtitia Marty(2), Olivier Sandre ${ }^{(3)}$ et Robert Whitney ${ }^{(4)}$

(1) Présidente de la SFP. (2) Présidente de la section Alpes de la SFP. (3) Président de la division Matière Condensée de la SFP. (4) Président du comité local d'organisation. 